\title{
Effects of Variable Viscosity and Thermal Conductivity on MHD Flow Past a Vertically Moving Porous Plate with Viscous and Joule Dissipation
}

\author{
G.C. Hazarika \\ Department of Mathematics, \\ Dibrugarh University, \\ Dibrugarh, Assam, India
}

\author{
Kalpana Goswami \\ Department of Mathematics, \\ Kherajkhat College, Lakhimpur, \\ Assam, India
}

\author{
Jadav Konch \\ Department of Mathematics, \\ Dibrugarh University, \\ Dibrugarh, Assam, India
}

\begin{abstract}
The effects of variable viscosity and thermal conductivity on a three-dimensional free convection flow past a uniformly moving porous vertical plate with viscous dissipation and Joule heating in presence of a uniform transverse magnetic field have been studied numerically. The governing boundary layer equations with associated boundary conditions are converted to non-dimensional form. The Magnetic Reynolds number is considered small enough to neglect the induced hydro magnetic effect. The resulting non-linear partial differential equations are then solved using an iterative method for an implicit finite difference scheme. Effects of various flow governing parameters on the fluid velocity, temperature, concentration fields are presented graphically. Further, the coefficient of skin-friction, rate of heat transfer and rate of mass transfer at the plate are presented in tabular form.
\end{abstract}

\section{Keywords}

Variable viscosity and thermal conductivity, Joule heating, viscous dissipation, MHD, free convection, mass and heat transfer.

\section{INTRODUCTION}

The study of the effects of a magnetic field on the flow of a viscous incompressible and electrically conducting fluid is important due to its practical application in many fields such as polymer production, nuclear technology, design and operation of many diverse forms of pre-heaters, exchangers coolers etc. Flow through porous media has attracted the attention of many researchers in fluid mechanics, because of its importance in the fields like extraction of the energy from geothermal regions.

Free convection flow past a vertical plate in the presence of transverse magnetic field has been studied by several researchers. Kim [1] has studied the magneto hydrodynamic convective heat transfer past a semi -infinite vertical porous moving plate with variable suction. Hazarika[2] has studied the effects of variable viscosity and thermal conductivity on MHD rotating fluid with dissipation and chemical reaction. Ahmed et al. [3] considers the effects of thermal diffusion on a three dimensional MHD free convection flow of a viscous incompressible fluid over a vertical plate embedded in a porous medium. Sharma and Mathur[4] investigated the steady laminar free convective flow of an electrically conducting fluid along a porous hot vertical infinite plate in the presence of a heat source or sink.
Hussain et al.[5] discussed the effects of radiation on free convection flow of a fluid with variable viscosity from a porous vertical plate. Seth et al.[6] studied the unsteady natural convection flow of a viscous incompressible electrically conducting fluid past an impulsively moving vertical plate in a porous medium with ramped wall temperature taking into account the effects of thermal radiation.

In most of the above studies were confined to fluid with constant viscosity and constant thermal conductivity. However it is known that these two physical properties may change significantly with temperature. Thus to predict the flow and heat transfer rate accurately it is important to consider the effects of temperature dependent viscosity and thermal conductivity variation

The purpose of the present work is to study the effects of variable viscosity and variable thermal conductivity on MHD free convection flow past a uniformly moving porous vertical plate with viscous dissipation and Joule heating.

\section{MATHEMATICAL FORMULATION}

The equations governing the steady motion of an incompressible viscous electrically conducting fluid with variable viscosity and thermal conductivity in presence of a magnetic field are:

Equation of continuity: $\operatorname{div} \vec{q}=0$

Gauss's law of magnetism: $\operatorname{div} \vec{B}=0$

Momentum equation:

$(\vec{q} \cdot \vec{\nabla}) \vec{q}=-\frac{1}{\rho} \vec{\nabla} p+\frac{\vec{J} \times \vec{B}}{\rho}+v \nabla^{2} \vec{q}+\vec{g}$

Energy equation:

$\rho c_{p}[(\vec{q} \cdot \vec{\nabla}) \bar{T}]=k \nabla^{2} \bar{T}+\varphi+\frac{\vec{J}^{2}}{\sigma}$

Species continuity equation:

$(\vec{q} \cdot \vec{\nabla}) \bar{C}=D_{m} \nabla^{2} \bar{C}+D_{T} \nabla^{2} \bar{T}$

Ohm's law: $\quad \vec{J}=\sigma[\vec{E}+\vec{q} \times \vec{B}]$ 
Let us consider a steady free convection flow of an incompressible viscous electrically conducting fluid passed a uniformly moving porous vertical plate with transverse sinusoidal suction velocity.

We now introduce a coordinate system $(\bar{X}, \bar{y}, \bar{Z})$ with $\bar{X}$-axis vertically upwards along the plate, $\bar{Y}$-axis perpendicular to it and directed into the fluid region and $\bar{Z}$-axis along the width of the plate is as shown in the Fig.1. (i) The fluid properties are assumed to be constant, except for the fluid viscosity and thermal conductivity which are assumed to vary as an inverse linear function of temperature.

(ii) A magnetic field of uniform strength $B_{o}$ is applied normal to the plate.

(iii) The magnetic Reynold's number small enough so that the induced magnetic field is negligible.

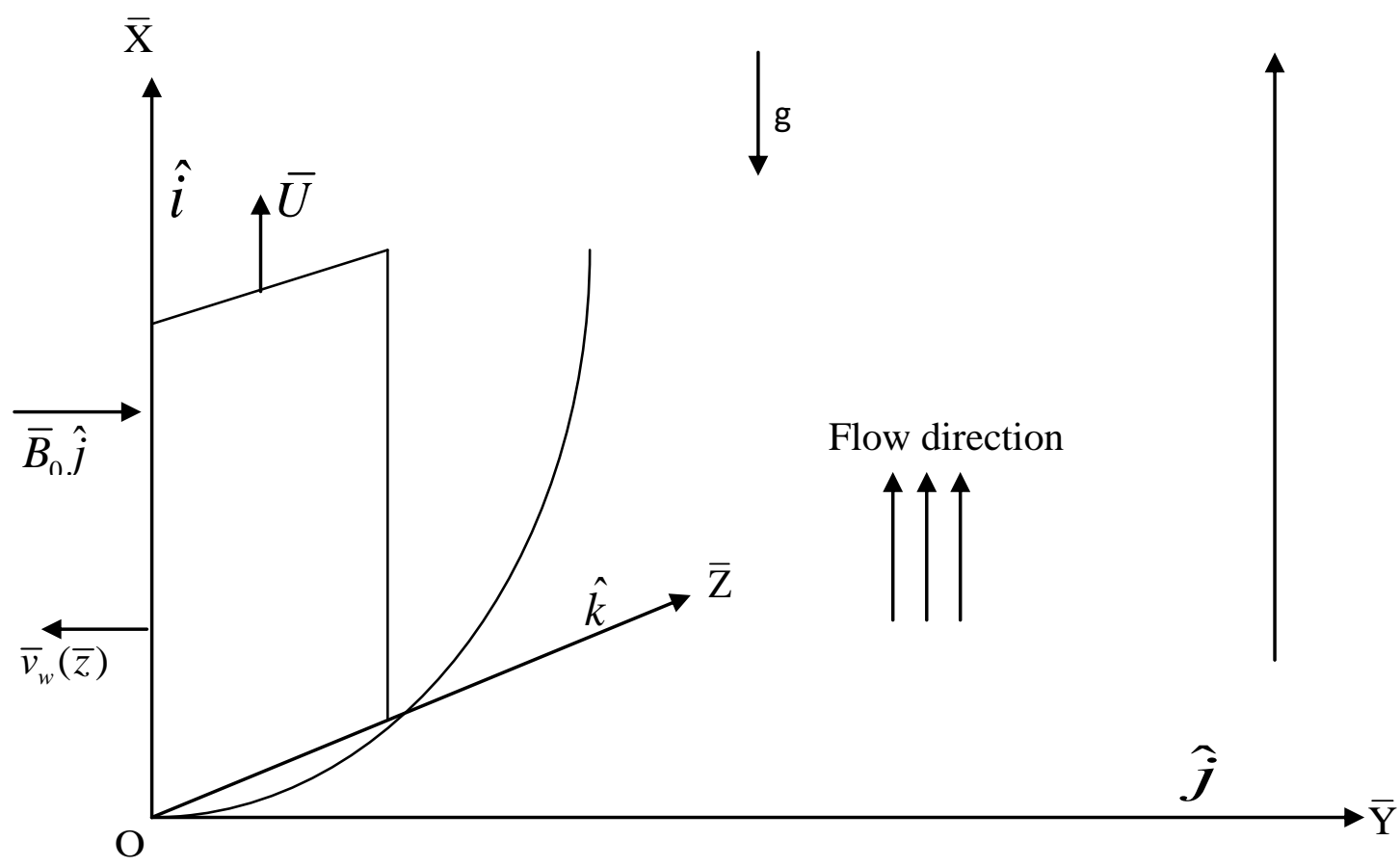

Fig. 1: Flow Configuration

Let $\vec{q}=\hat{i} \bar{u}+\hat{j} \bar{v}+\hat{k} \bar{w}$ be the fluid velocity at the point $(\bar{x}, \bar{y}, \bar{z}), \vec{U}=\bar{U} \hat{i}$ be the plate velocity and $\vec{B}=B_{0}$

(iv) The viscous dissipation and magnetic dissipation of energy are considered. $\hat{j}$ be the applied magnetic field, $\hat{i}, \hat{j}, \hat{k}$ being the unit (v) The pate is electrically non-conducting. vectors along positive direction of $\bar{X}$-axis, $\bar{Y}$-axis and $\bar{Z}$-axis respectively.

The suction velocity is taken as

$$
\bar{v}_{w}(\bar{z})=-V_{0}\left[1+\varepsilon \cos \frac{\pi \bar{z}}{L}\right]
$$

where, $\varepsilon$ is small reference parameter such that $\varepsilon \square 1$ and $L$ is the wave length of the periodic suction

Following assumptions were made for our present study:

$$
\frac{\partial \bar{v}}{\partial \bar{y}}+\frac{\partial \bar{w}}{\partial \bar{z}}=0
$$


Momentum equations:

$$
\begin{gathered}
\rho\left[\bar{v} \frac{\partial \bar{u}}{\partial \bar{y}}+\bar{w} \frac{\partial \bar{u}}{\partial \bar{z}}\right]=\rho g \beta\left(\bar{T}-\overline{T_{\propto}}\right)+\rho g \bar{\beta}\left(\bar{c}-\overline{c_{\alpha}}\right) \\
+\left[\frac{\partial \mu}{\partial \bar{y}} \frac{\partial \bar{u}}{\partial \bar{y}}+\frac{\partial \mu}{\partial \bar{z}} \frac{\partial \bar{u}}{\partial \bar{z}}\right] \\
+\mu\left[\frac{\partial^{2} \bar{u}}{\partial \bar{y}^{2}}+\frac{\partial^{2} \bar{u}}{\partial \bar{z}^{2}}\right]-\sigma B_{0}^{2} \bar{u} \\
\rho\left[\bar{v} \frac{\partial \bar{v}}{\partial \bar{y}}+\bar{w} \frac{\partial \bar{v}}{\partial \bar{w}}\right]=-\frac{\partial \bar{p}}{\partial \bar{y}}+\left[\frac{\partial \mu}{\partial \bar{y}} \frac{\partial \bar{v}}{\partial \bar{y}}+\frac{\partial \mu}{\partial \bar{z}} \frac{\partial \bar{v}}{\partial \bar{z}}\right]+\mu\left[\frac{\partial^{2} \bar{v}}{\partial \bar{y}^{2}}+\frac{\partial^{2} \bar{v}}{\partial \bar{z}^{2}}\right] \\
\rho\left[\bar{v} \frac{\partial \bar{w}}{\partial \bar{y}}+\bar{w} \frac{\partial \bar{w}}{\partial \bar{z}}\right]=-\frac{\partial \bar{p}}{\partial \bar{z}}+\left[\frac{\partial \mu}{\partial \bar{y}} \frac{\partial \bar{w}}{\partial \bar{y}}+\frac{\partial \mu}{\partial \bar{z}} \frac{\partial \bar{w}}{\partial \bar{z}}\right] \\
+\mu\left[\frac{\partial^{2} \bar{w}}{\partial \bar{y}^{2}}+\frac{\partial^{2} \bar{w}}{\partial \bar{z}^{2}}\right]-\sigma B_{0}^{2} \bar{w}
\end{gathered}
$$

The energy equation:

$$
\begin{aligned}
\rho C_{p}\left[\bar{v} \frac{\partial \bar{T}}{\partial \bar{y}}+\bar{w}\right. & \left.\frac{\partial \bar{T}}{\partial \bar{z}}\right] \\
& =\left[\frac{\partial k}{\partial \bar{y}} \frac{\partial \bar{T}}{\partial \bar{y}}+\frac{\partial k}{\partial \bar{z}} \frac{\partial \bar{T}}{\partial \bar{z}}\right] \\
& +k\left[\frac{\partial^{2} \bar{T}}{\partial \bar{y}^{2}}+\frac{\partial^{2} \bar{T}}{\partial \bar{z}^{2}}\right] \\
+2 \mu\left[\left(\frac{\partial \bar{v}}{\partial \bar{y}}\right)^{2}+\right. & \left.\left(\frac{\partial \bar{w}}{\partial \bar{z}}\right)^{2}\right] \\
& +\mu\left[\left(\frac{\partial \bar{u}}{\partial \bar{y}}\right)^{2}+\left(\frac{\partial \bar{u}}{\partial \bar{z}}\right)^{2}\right. \\
& \left.+\left(\frac{\partial \bar{w}}{\partial \bar{y}}+\frac{\partial \bar{v}}{\partial \bar{z}}\right)^{2}\right]
\end{aligned}
$$$$
+\sigma\left(\bar{u}^{2}+\bar{v}^{2}+\bar{w}^{2}\right) B_{0}^{2}
$$

Species continuity equation:

$$
\begin{aligned}
\bar{v} \frac{\partial \bar{c}}{\partial \bar{y}}+\bar{w} \frac{\partial \bar{c}}{\partial \bar{z}}= & \frac{\partial}{\partial \bar{y}}\left(D_{M} \frac{\partial \bar{c}}{\partial \bar{y}}\right)+\frac{\partial}{\partial \bar{z}}\left(D_{M} \frac{\partial \bar{c}}{\partial \bar{z}}\right) \\
& +\frac{\partial}{\partial \bar{y}}\left(D_{T} \frac{\partial}{\partial \bar{y}} \bar{T}\right) \\
& +\frac{\partial}{\partial \bar{z}}\left(D_{T} \frac{\partial}{\partial \bar{z}} \bar{T}\right)
\end{aligned}
$$

The relevant boundary conditions are:

$$
\begin{aligned}
& \bar{y}=0: \bar{u}=\bar{U}, \bar{v}=\bar{v}_{w}, \bar{w}=0, \bar{T}=\bar{T}_{w}, \bar{c}=\bar{c}_{w} \\
& \bar{y} \rightarrow \propto: \bar{u}=0, \bar{v}=-V_{0}, \bar{w}=0, \bar{T}=\bar{T}_{\alpha}, \bar{c}=\bar{c}_{\propto}, \bar{p}=\bar{p}_{\propto}
\end{aligned}
$$

We introduce the following non-dimensional variable and parameters:

$$
\begin{aligned}
& y=\frac{\bar{y}}{L}, z=\frac{\bar{z}}{L}, u=\frac{\bar{u}}{V_{0}}, v=\frac{\bar{v}}{V_{0}}, w=\frac{\bar{w}}{V_{0}}, U=\frac{\bar{U}}{V_{0}}, \theta=\frac{\bar{T}-\bar{T}_{\alpha}}{\bar{T}_{w}-\bar{T}_{\alpha}}, \\
& \varphi=\frac{\bar{c}-\bar{c}_{\propto}}{\bar{c}_{w}-\bar{c}_{\propto}}, P=\frac{v}{\alpha}, S_{c}=\frac{v}{D_{M}}, S_{0}=\frac{D_{T}\left(\bar{T}_{w}-\bar{T}_{\propto}\right)}{v\left(\bar{c}_{w}-\bar{c}_{\propto}\right)}, \\
& G_{r}=\frac{\operatorname{Lg} \beta\left(\bar{T}_{w}-\bar{T}_{\propto}\right)}{V_{0}^{2}}, G_{M}=\frac{\operatorname{Lg} \bar{\beta}\left(\bar{c}_{w}-\bar{c}_{\propto}\right)}{V_{0}^{2}}, M=\frac{\sigma B_{0}^{2} v}{\rho V_{0}^{2}}, R e \\
& =\frac{V_{0} L}{v} \\
& p_{\propto}=\frac{\bar{p}_{\propto}}{\rho\left(\frac{v}{L}\right)^{2}}, E_{c}=\frac{V_{0}^{2}}{C_{p}\left(\bar{T}_{w}-\bar{T}_{\propto}\right)}
\end{aligned}
$$

Here $\mu, \mathrm{k}, \sigma$ are the viscosity, thermal conductivity and electrical conductivity of the fluid respectively.

Lai and Kulacki[7] have assumed the viscosity of the fluid to be inverse linear function of the temperature as ,

$$
\begin{aligned}
& \frac{1}{\mu}=\frac{1}{\mu_{\propto}}\left[1+\alpha\left(\bar{T}-\bar{T}_{\propto}\right)\right] \\
& \text { Or, } \quad \frac{1}{\mu}=b\left(\bar{T}-\bar{T}_{r}\right)
\end{aligned}
$$

Or, $\mu=\frac{1}{b\left(\bar{T}-\bar{T}_{r}\right)}$, where $\mathrm{b}=\frac{\alpha}{\mu_{\alpha}}, \bar{T}_{r}=\bar{T}_{\propto}-\frac{1}{\alpha}$, where $\mu_{\propto}$ is the free stream viscosity, $\mathrm{b}$ and $\bar{T}_{\propto}$ are constants whose values depend upon the reference state and thermal property of the fluid, $\bar{T}_{r}$ is transformed reference temperature related to viscosity parameter, $\alpha$ is a constant based on the thermal property of the fluid and $b<0$ for gas and $b>0$ for liquid.

In the light of Lai and Kulacki[7] and following Hazarika and Khound[8], let us consider the variation of thermal conductivity as,

$$
\begin{aligned}
& \frac{1}{k}=\frac{1}{k_{\propto}}\left[1+\beta\left(\bar{T}-\bar{T}_{\propto}\right)\right] \\
& \text { or, } k=\frac{1}{c\left(\overline{\bar{T}}-\bar{T}_{c}\right)}, \quad \text { where } \quad \bar{c}=\frac{\beta}{k_{\alpha}}, \text { and } \bar{T}_{c}=\bar{T}_{\propto}-\frac{1}{\beta^{\prime}}
\end{aligned}
$$

where $\mathrm{c}$ and $\bar{T}_{c}$ are constants and their values depend upon the reference state and thermal properties of the fluid i.e. $\beta$.

Thus the non-dimensional forms of equations are:

The equation of continuity:

$$
\frac{\partial v}{\partial y}+\frac{\partial w}{\partial z}=0
$$

The momentum equations:

$$
\begin{aligned}
& v \frac{\partial u}{\partial y}+w \frac{\partial u}{\partial z}=G_{r} \theta+G_{m} \varphi+\frac{1}{R e(} \frac{\theta_{r}}{\left.\theta-\theta_{r}\right)^{2}}\left[\frac{\partial \theta}{\partial y} \frac{\partial u}{\partial y}+\frac{\partial \theta}{\partial z} \frac{\partial u}{\partial z}\right] \\
& -\frac{1}{\operatorname{Re}\left(\theta-\theta_{r}\right)}\left[\frac{\partial^{2} u}{\partial y^{2}}+\frac{\partial^{2} u}{\partial z^{2}}\right]-M R e u
\end{aligned}
$$

$$
v \frac{\partial v}{\partial y}+w \frac{\partial v}{\partial z}=-\frac{1}{R e^{2}}\left(\frac{\theta_{r}}{\theta-\theta_{r}}\right)^{2} \frac{\partial p}{\partial y}+\frac{2 p}{R e^{2}} \frac{\theta_{r}^{2}}{\left(\theta-\theta_{r}\right)^{3}} \frac{\partial \theta}{\partial y}
$$




$$
\begin{aligned}
& +\frac{1}{R e} \cdot \frac{\theta_{r}}{\left(\theta-\theta_{r}\right)^{2}}\left[\frac{\partial \theta}{\partial y} \frac{\partial v}{\partial y}+\frac{\partial \theta}{\partial z} \frac{\partial v}{\partial z}\right]-\frac{1}{R e}\left(\frac{\theta_{r}}{\theta-\theta_{r}}\right) \cdot\left[\frac{\partial^{2} v}{\partial y^{2}} \frac{\partial^{2} v}{\partial z^{2}}\right] \\
& v \frac{\partial w}{\partial y}+w \frac{\partial w}{\partial z}=-\frac{1}{R e^{2}}\left(\frac{\theta_{r}}{\theta-\theta_{r}}\right)^{2} \frac{\partial p}{\partial z}+ \\
& \frac{2 p}{R e^{2}} \frac{\theta_{r}^{2}}{\left(\theta-\theta_{r}\right)^{3}} \frac{\partial \theta}{\partial z} \\
& +\frac{1}{R e} \cdot \frac{\theta_{r}}{\left(\theta-\theta_{r}\right)^{2}}\left[\frac{\partial \theta}{\partial y} \frac{\partial w}{\partial y}+\frac{\partial \theta}{\partial z} \frac{\partial w}{\partial z}\right]-\frac{1}{R e}\left(\frac{\theta_{r}}{\theta-\theta_{r}}\right) \cdot\left[\frac{\partial^{2} w}{\partial y^{2}}+\frac{\partial^{2} w}{\partial z^{2}}\right]- \\
& \text { MRew } \\
& v \frac{\partial \theta}{\partial y}+w \frac{\partial \theta}{\partial z}= \\
& \frac{1}{P R e} \cdot \frac{\theta_{c}}{\left(\theta-\theta_{c}\right)^{2}}\left[\left(\frac{\partial \theta}{\partial y}\right)^{2}+\left(\frac{\partial \theta}{\partial z}\right)^{2}\right]-\frac{1}{P R e}\left(\frac{\theta_{c}}{\theta-\theta_{c}}\right)\left[\frac{\partial^{2} \theta}{\partial y^{2}}+\frac{\partial^{2} \theta}{\partial z^{2}}\right] \\
& -2 \frac{E_{c}}{R e}\left(\frac{\theta_{r}}{\theta-\theta_{r}}\right)\left[\left(\frac{\partial v}{\partial y}\right)^{2}+\left(\frac{\partial w}{\partial z}\right)^{2}\right]-\frac{E_{c}}{R e}\left(\frac{\theta_{r}}{\theta-\theta_{r}}\right) \\
& \times\left[\left(\frac{\partial u}{\partial y}\right)^{2}+\left(\frac{\partial u}{\partial z}\right)^{2}+\left(\frac{\partial w}{\partial y}+\frac{\partial v}{\partial z}\right)^{2}\right]+\frac{M E_{c}}{R e}\left(u^{2}+w^{2}\right)
\end{aligned}
$$

The species concentration equation:

$$
\begin{gathered}
v \frac{\partial \varphi}{\partial y}+w \frac{\partial \varphi}{\partial z}=\frac{1}{S_{c} \operatorname{Re}} \frac{\theta_{r}}{\left(\theta-\theta_{r}\right)^{2}}\left[\frac{\partial \theta}{\partial y} \frac{\partial \varphi}{\partial y}+\frac{\partial \theta}{\partial z} \frac{\partial \varphi}{\partial z}\right] \\
-\frac{1}{S_{c} \operatorname{Re} \frac{\theta_{r}}{\left(\theta-\theta_{r}\right)}} \\
x\left[\frac{\partial^{2} \varphi}{\partial y^{2}}+\frac{\partial^{2} \varphi}{\partial z^{2}}\right]+\frac{S_{0}}{R e} \frac{\theta_{r}}{\left(\theta-\theta_{r}\right)^{2}} \cdot\left[\left(\frac{\partial \theta}{\partial y}\right)^{2}+\left(\frac{\partial \theta}{\partial z}\right)^{2}\right] \\
-\frac{S_{0}}{R e} \frac{\theta_{r}}{\left(\theta-\theta_{r}\right)} \cdot\left[\frac{\partial^{2} \theta}{\partial y^{2}}+\frac{\partial^{2} \theta}{\partial z^{2}}\right]
\end{gathered}
$$

The relevant boundary conditions are:

$\left.\begin{array}{l}\text { i.e; } \quad \mathrm{y}=0: \mathrm{u}=\mathrm{U}, \quad \mathrm{v}=-(1+\mathcal{E}(\cos \pi \mathrm{z})), \mathrm{w}=0, \theta=1, \varphi=1 \\ \mathrm{y} \rightarrow \infty: \mathrm{u}=0, \quad \mathrm{v}=-1, \quad \mathrm{w}=0, \theta=0, \varphi=0, \quad \mathrm{P}=P_{\infty}\end{array}\right\}$

In this problem, the physical quantities of engineering interest are the coefficient of skin- friction $\left(\mathrm{C}_{\mathrm{f}}\right)$, Nusselt number $(\mathrm{Nu})$ and Sherwood number (Sh), which are as below:

\subsection{The coefficient of skin-friction:}

The viscous drag at the plate per unit area in the direction of the plate velocity is given by the Newton's law of viscosity in the form:

$$
\left.\tau^{\prime}=-\mu \frac{\partial \bar{u}}{\partial \bar{y}}\right]_{\bar{y}=0}=\left[-\frac{\mu V_{0}}{L} \frac{\partial u}{\partial y}\right]_{y=0}
$$

The non dimensional skin friction at the plate in the direction of the free stream is given by,

$$
\begin{aligned}
C_{f} & =-\frac{\left.-\mu \frac{\partial \bar{u}}{\partial \bar{y}}\right]_{\bar{y}=0}}{\rho\left(V_{0}\right)^{2}} \\
& =\left[-\frac{1}{R e} \frac{\partial u}{\partial y}\right]_{y=0}
\end{aligned}
$$

\subsection{The Nusselt number:}

The Nusselt number measures the rate of heat transfer at the plate.

The heat flux q from the plate to the fluid is given by the Fourier's law of heat conduction in the form

$$
\begin{aligned}
\mathrm{q} & =\left[-K \frac{\partial \bar{T}}{\partial \bar{y}}\right]_{\bar{y}=0} \\
& =\left[\frac{-K\left(\bar{T}_{w}-\bar{T}_{\infty}\right)}{L} \frac{\partial \theta}{\partial y}\right]_{y=0}
\end{aligned}
$$

The rate of heat transfer from the plate to the fluid in terms of Nusselt Number is given by

$$
\begin{aligned}
N_{u} & =-\frac{K}{\rho V_{0} C_{p}\left(\bar{T}_{w}-\bar{T}_{\infty}\right)}\left[\frac{\partial \bar{T}}{\partial \bar{y}}\right]_{\bar{y}=0} \\
& =\left[-\frac{1}{P R e} \frac{\partial \theta}{\partial y}\right]_{y=0}
\end{aligned}
$$

\subsection{Sherwood number:}

The Sherwood number measures the rate of mass transfer at the plate and is given by

$$
\begin{gathered}
S_{h}=\frac{D_{M}}{V_{0}\left(\overline{C_{w}}-\overline{C_{\infty}}\right)}\left(\frac{\partial \bar{c}}{\partial \bar{y}}\right)_{y=0} \\
=\left[-\frac{1}{S_{c} R e} \frac{\partial \phi}{\partial y}\right]_{y=0}
\end{gathered}
$$

\section{METHOD OF SOLUTION}

The boundary value problem (18)-(24) is discritized with ordinary finite difference approximations and reduced to a system of difference equations. In this technique derivatives occurring in the generated differential equations have been replaced by their finite difference approximation. The approximation for an independent variable ' $\mathrm{f}$ ' is given by

$$
\begin{aligned}
& \frac{\partial f}{\partial x}=\frac{f(i+1, j)-f(i, j)}{\Delta x} \\
& \frac{\partial^{2} f}{\partial y^{2}}=\frac{f(i, j+1)-2 f(i, j)+f(i, j-1)}{\Delta y^{2}}
\end{aligned}
$$

Using these formula in (18) to (23) and after simplification we get the following implicit schemes for velocity, temperature, pressure and species concentration viz., u, v, w, $\Theta, \mathrm{p}$ and $\varphi$.

$$
v(i, j)=v(i+1, j)+\frac{h}{k}[w(i, j+1)-w(i, j)]
$$

$u(i, j)=\left[-\frac{v(i, j) \cdot u(i+1, j)}{h}-\frac{w(i, j) \cdot u(i, j+1)}{k}+\operatorname{Gr} \theta(i, j)+\operatorname{Gm} \varphi(i, j)\right.$ 


$$
\begin{aligned}
& +\frac{\theta_{r}}{\operatorname{Re}\left\{\theta(i, j)-\theta_{r}\right\}^{2}}\left\{u(i+1, j) \cdot \frac{\theta(i+1, j)-\theta(i, j)}{h^{2}}\right. \\
& \left.+u(i, j+1) \cdot \frac{\theta(i, j+1)-\theta(i, j)}{k^{2}}\right\}-\frac{\theta_{r}}{\operatorname{Re}\left\{\theta(i, j)-\theta_{r}\right\}}\left\{\frac{\theta(i+1, j)-u(i-1, j)}{h^{2}}\right. \\
& \left.\left.+\frac{u(i, j+1)-u(i, j-1)}{k^{2}}\right\}\right] /\left[-\frac{v(i, j)}{h}-\frac{w(i, j)}{k}+\frac{\theta_{r}}{\operatorname{Re}\left(\theta(i, j)-\theta_{r}\right)^{2}}\right. \\
& \left.\times\left[\frac{\theta(i+1, j)-\theta(i, j)}{h^{2}}+\frac{\theta(i, j+1)-\theta(i, j)}{k^{2}}\right]-\frac{2 \theta_{r}}{\operatorname{Re}\left(\theta(i, j)-\theta_{r}\right)}\left(\frac{1}{h^{2}}+\frac{1}{k^{2}}+M \operatorname{Re}\right)\right]
\end{aligned}
$$

$p(i, j)=\left[-\frac{1}{\operatorname{Re}^{2}}\left(\frac{\theta_{r}}{\theta-\theta_{r}}\right)^{2} \frac{p(i+1, j)}{h}+v(i, j) \frac{v(i+1, j)-v(i, j)}{h}\right.$

$+\frac{2 P}{\operatorname{Re}^{2}} \frac{\theta_{r}^{2}}{\left(\theta(i, j)-\theta_{r}\right)^{3}} \frac{\theta(i+1, j)-\theta(i, j)}{h}+\frac{1}{\operatorname{Re}} \frac{\theta}{\left(\theta(i, j)-\theta_{r}\right)^{2}}\left[\frac{\theta(i+1, j)-\theta(i, j)}{h} \frac{v(i+1, j)-v(i, j)}{h}\right.$

$\left.+\frac{\theta(i, j+1)-\theta(i, j)}{k} \frac{v(i, j+1)-v(i, j)}{k}\right]-\frac{1}{\operatorname{Re}} \frac{\theta_{r}}{\theta-\theta_{r}}\left[\frac{v(i+1, j)-2 v(i, j)+v(i-1, j)}{h^{2}}\right.$ $\left.\left.+\frac{v(i, j+1)-2 v(i, j)+v(i, j-1)}{k^{2}}\right]\right] /\left[-\frac{1}{h \operatorname{Re}^{2}}\left(\frac{\theta_{r}}{\theta(i, j)-\theta_{r}}\right)^{2}\right]$

$w(i, j)=\left[-\frac{v(i, j)^{*} w(i, j)}{h}-\left\{\frac{1}{\operatorname{Re}^{2}} \frac{\theta_{r}}{\theta(i, j)-\theta_{r}}\right\}^{2} \frac{p(i, j+1)-p(i, j)}{k}\right.$ $+\frac{2 P}{\operatorname{Re}^{2}} \frac{\theta_{r}^{2}}{\left(\theta(i, j)-\theta_{r}\right)^{3}} \frac{\theta(i, j+1)-\theta(i, j)}{k}+\frac{1}{\operatorname{Re}} \frac{\theta_{r}}{\left(\theta(i, j)-\theta_{r}\right)^{2}}\left\{\frac{\theta(i+1, j)-\theta(i, j)}{h^{2}} w(i+1, j)\right.$ $\left.+\frac{\theta(i, j+1)-\theta(i, j)}{h^{2}} w(i, j+1)\right\}-\frac{1}{\operatorname{Re}} \frac{\theta_{r}}{\theta(i, j)-\theta_{r}}\left\{\frac{w(i+1, j)+w(i, j)}{h^{2}}\right.$ $\left.\left.+\frac{w(i, j+1)+w(i, j)}{k^{2}}\right\}\right] /\left[-\frac{v(i, j)}{h}+\frac{w(i, j+1)-w(i, j)}{k}-\frac{1}{\operatorname{Re}} \frac{\theta_{r}}{\left(\theta(i, j)-\theta_{r}\right)^{2}}\right.$ $\left.\times\left\{-\frac{\theta(i+1, j)-\theta(i, j)}{h^{2}}-\frac{\theta(i, j+1)-\theta(i, j)}{k^{2}}\right\}-\frac{2}{\operatorname{Re}} \frac{\theta_{r}}{\left(\theta(i, j)-\theta_{r}\right)}\left(\frac{1}{h^{2}}+\frac{1}{k^{2}}\right)+M \operatorname{Re}\right]$

$\theta(i, j)=\left[-\frac{v(i, j) * \theta(i+1, j)}{h}-\frac{w(i, j) * \theta(i, j+1)}{h}-\frac{1}{P \operatorname{Re}} \frac{\theta_{c}}{\left(\theta(i, j)-\theta_{c}\right)^{2}}\right.$

$\times\left[\left\{\frac{\theta(i+1, j)}{h}\right\}^{2}+\left\{\frac{\theta(i, j+1)}{k}\right\}^{2}\right]-\frac{1}{P \operatorname{Re}} \frac{\theta_{c}}{\left(\theta(i, j)-\theta_{c}\right)}\left\{\frac{\theta(i+1, j)+\theta(i-1, j)}{h^{2}}+\frac{\theta(i, j+1)+\theta(i, j-1)}{k^{2}}\right\}$ $-\frac{2 E c}{\operatorname{Re}} \frac{\theta_{r}}{\theta(i, j)-\theta_{r}}\left\{\left(\frac{w(i+1, j)+w(i, j)}{h}\right)^{2}+\left(\frac{w(i, j+1)+w(i, j)}{k}\right)^{2}\right\}$

$-\frac{E c}{\operatorname{Re}} \frac{\theta_{r}}{\left(\theta(i, j)-\theta_{r}\right)}\left[\left\{\frac{u(i+1, j)-u(i, j)}{h}\right\}^{2}+\left\{\frac{u(i, j-1)-u(i, j)}{k^{2}}\right\}^{2}\right.$ $\left.+\left\{\frac{w(i+1, j)-w(i, j)}{h}+\frac{v(i, j+1)-v(i, j)}{k}\right\}^{2}\right]-\frac{M E c}{\operatorname{Re}}\left[\{u(i, j)\}^{2}\right.$ $\left.\left.+\{w(i, j)\}^{2}\right]\right] /\left[-\frac{v(i, j)}{h}-\frac{w(i, j)}{k}+\frac{2}{P \operatorname{Re}} \frac{\theta_{c}}{\left(\theta(i, j)-\theta_{c}\right)^{2}}\right.$ $\left.\times\left\{\frac{\theta(i+1, j)+\theta(i, j)}{h^{2}}+\frac{\theta(i, j+1)+\theta(i, j)}{k^{2}}\right\}-\frac{2}{P \operatorname{Re}} \frac{\theta_{c}}{\theta(i, j)-\theta_{c}}\left(\frac{1}{h^{2}}+\frac{1}{k^{2}}\right)\right]$

$\varphi(i, j)=\left[-\frac{v(i, j) \cdot \varphi(i+1, j)}{h}-\frac{w(i, j) \cdot \varphi(i, j+1)}{k}+\frac{\theta_{r}}{S c \operatorname{Re}\left\{\theta(i, j)-\theta_{r}\right\}^{2}}\right.$

$\times\left\{\varphi(i+1, j) \cdot \frac{\theta(i+1, j)-\theta(i, j)}{h^{2}}-\frac{1}{S c \operatorname{Re}} \frac{\theta_{r}}{\left\{\theta(i, j)-\theta_{r}\right\}}\left\{\frac{\varphi(i+1, j)-\varphi(i-1, j)}{h^{2}}\right.\right.$ $\left.+\frac{\varphi(i, j+1)-\varphi(i, j-1)}{k^{2}}\right\}+\frac{S o}{\operatorname{Re}} \frac{\theta_{r}}{\left\{\theta(i, j)-\theta_{r}\right\}^{2}}\left[\left\{\frac{\theta(i+1, j)-\theta(i-1, j)}{h}\right\}^{2}\right.$

$\left.+\left\{\frac{\theta(i+1, j)-\theta(i-1, j)}{k}\right\}^{2}\right]-\frac{S o}{\operatorname{Re}} \frac{\theta_{r}}{\left\{\theta(i, j)-\theta_{r}\right\}}\left\{\frac{\theta(i+1, j)-2 \theta(i, j)+\theta(i-1, j)}{h^{2}}\right.$ $\left.\left.+\frac{\theta(i, j+1)-2 \theta(i, j)+\theta(i, j-1)}{h^{2}}\right\}\right] /\left[-\frac{v(i, j)}{h}-\frac{w(i, j)}{k}+\frac{1}{S c \operatorname{Re}} \frac{\theta_{r}}{\left(\theta(i, j)-\theta_{r}\right)^{2}}\right.$ $\left.\times\left\{\frac{\theta(i+1, j)-\theta(i, j)}{h^{2}}+\frac{\theta(i, j+1)-\theta(i, j)}{k^{2}}\right\}-\frac{2}{S c \operatorname{Re}} \frac{\theta_{r}}{\left(\theta(i, j)-\theta_{r}\right)}\left(\frac{1}{h^{2}}+\frac{1}{k^{2}}\right)\right]$

where $h=\Delta y$ and $k=\Delta z$.

Boundary conditions (24) are reduced to:

$\mathrm{u}(1, \mathrm{j})=\mathrm{U} ; \quad \mathrm{v}(1, \mathrm{j})=-\left(1+\mathrm{ep} * \cos \left(\mathrm{pi}^{*}(\mathrm{c}+(\mathrm{j}-1) * \mathrm{k})\right)\right) ; \mathrm{w}(1, \mathrm{j})=0 ;$ $\Theta(1, \mathrm{j})=1 ; \quad \Phi(1, \mathrm{j})=1 ; \quad \mathrm{u}(\mathrm{n}+1, \mathrm{j})=0 ; \quad \mathrm{v}(\mathrm{n}+1, \mathrm{j})=-1 ;$ $\mathrm{w}(\mathrm{n}+1, \mathrm{j})=0 ; \quad \Theta(\mathrm{n}+1, \mathrm{j})=0 ; \quad \Phi(\mathrm{n}+1, \mathrm{j})=0 ; \quad \mathrm{p}(\mathrm{n}+1, \mathrm{j})=\mathrm{P}_{\infty}$

The above systems of difference equations are then solved numerically by an iterative scheme. 


\section{RESULTS AND DISCUSSION}

Numerical solutions are obtained for the implicit schemes (25)-(30) together with (31) using an iterative scheme for the velocity field, temperature field and species concentration field. The coefficient of skin-friction, rate of heat transfer in terms of Nusselt number and the rate of mass transfer in terms of Sherwood number have been calculated by assigning some arbitrarily chosen specific values to the physical parameters involved in the problem viz., $\Theta_{\mathrm{r}}=-5, \Theta_{\mathrm{c}}=-4, \mathrm{Ec}=0.05, \mathrm{Gr}=0.2$ $5, \mathrm{Gm}=0.25, \mathrm{Pr}=2.71, \mathrm{Re}=0.75, \mathrm{Sc}=.22, \mathrm{Sr}=1, \mathrm{M}=1$, $\varepsilon=0.01, \Omega=2.5, \mathrm{z}=0.4$ unless otherwise stated.

Fig.2 to Fig.5 show the effects of viscosity parameter $\left(\Theta_{\mathrm{r}}\right)$, thermal conductivity parameter $\left(\Theta_{c}\right)$, Hartmann number(M) and Eckert number(Ec) on velocity field. From Fig.2 and Fig.4, it is observed that the velocity considerably decreased with the increase of $\Theta_{\mathrm{r}}$ and $M$. Because velocity decreases with the increase of viscosity and also due to the effect of Lorentz force one resistive term appears in the momentum equation which opposes the velocity of the fluid. From Fig. 3 and Fig.5, it is seen that velocity increases for increasing values of $\Theta_{c}$ and $\mathrm{Ec}$.

The temperature profiles are given in Fig.6 to Fig.11 for the parameters namely the viscosity parameter $\left(\theta_{r}\right)$, thermal conductivity parameter $\left(\theta_{c}\right)$, dissipation parameter $\left(E_{c}\right)$, Hartmann number $(\mathrm{M})$, Reynolds number(Re) and Prandtl number $\left(P_{r}\right)$. From these figures it is observed that the temperature decreases with the increase of $\theta_{r}, \mathrm{Ec}$ and Re; while that of increases with the increase of $\Theta_{c}, M$ and $P r$.

The species concentration profiles are presented in Fig.12 and Fig.13 for different values of Schimdt number $\left(S_{c}\right)$ and Soret number $\left(S_{o}\right)$. It is observed from these graphs that species concentration increases for the increasing values of Schmidt number and Soret number.

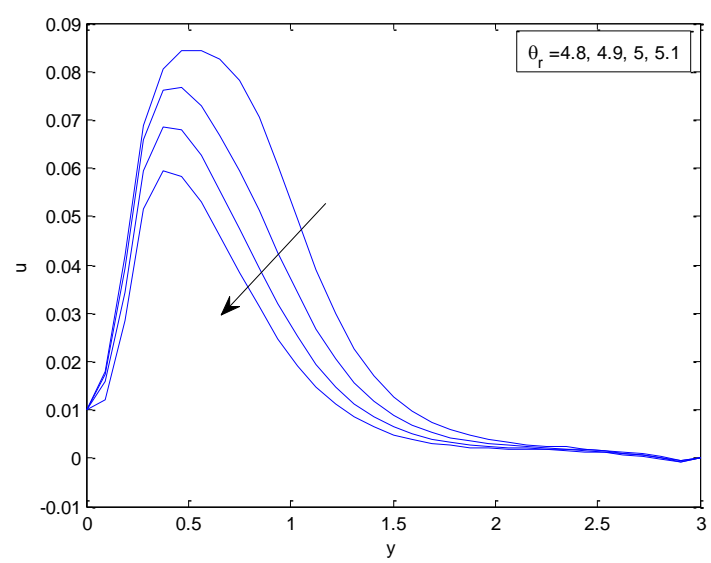

Fig.2: Velocity profile for different $\Theta_{\mathrm{r}}$

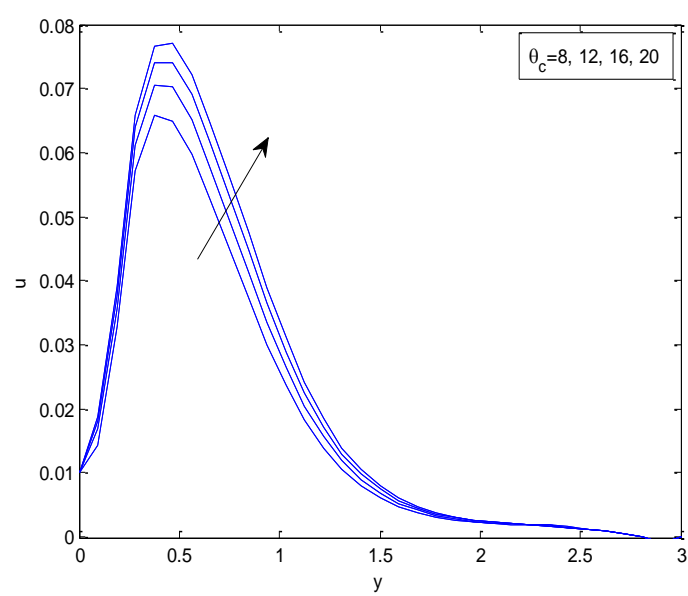

Fig.3: Velocity profile for different $\Theta_{c}$

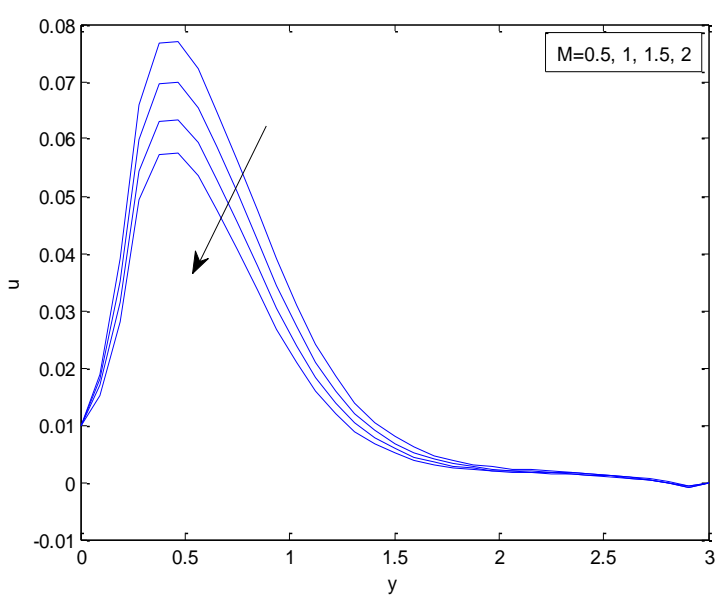

Fig.4: Velocity profile for different $M$

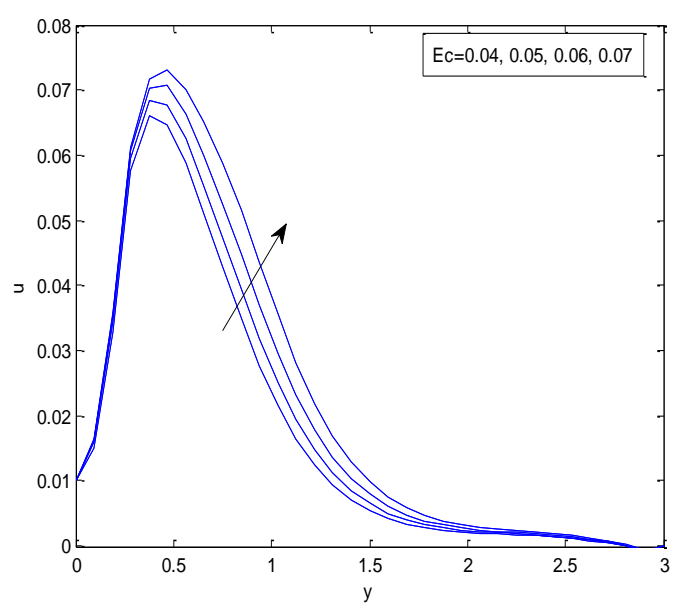

Fig.5: Velocity profile for different Ec 


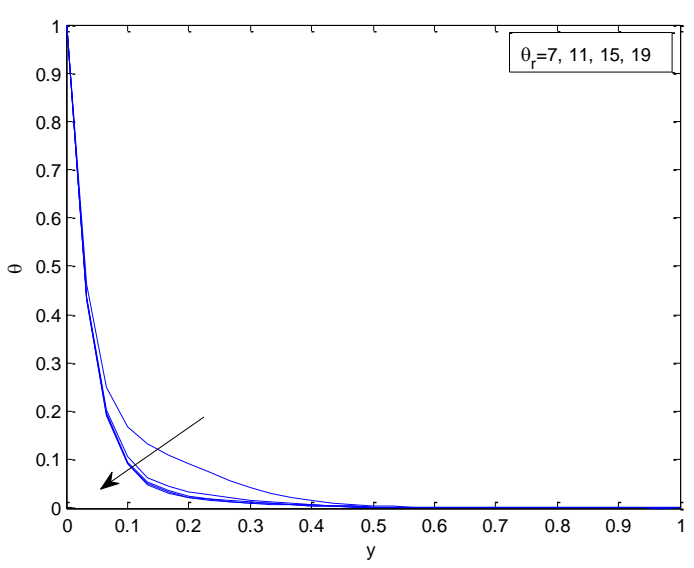

Fig.6: Temperature profile for different $\theta_{\mathrm{r}}$

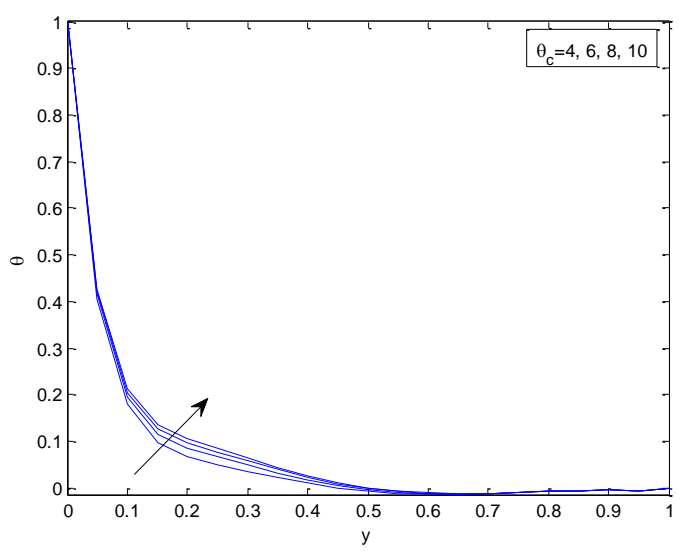

Fig.7: Temperature profile for different $\Theta_{c}$

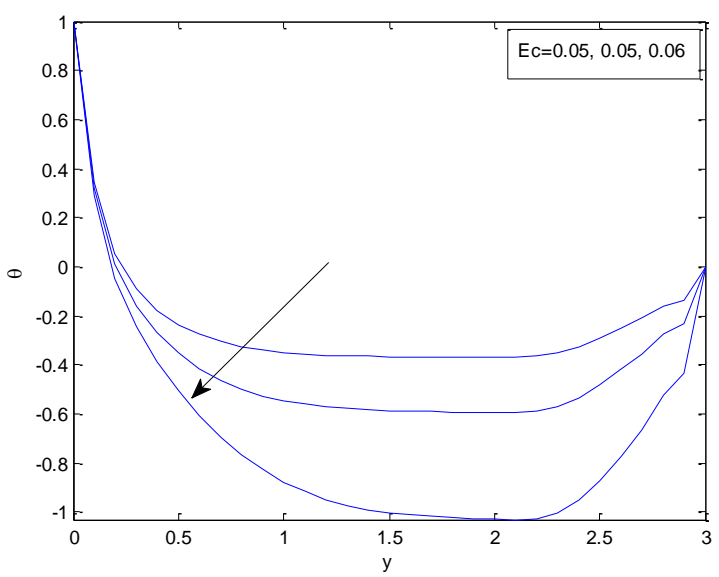

Fig.8: Temperature profile for different Ec

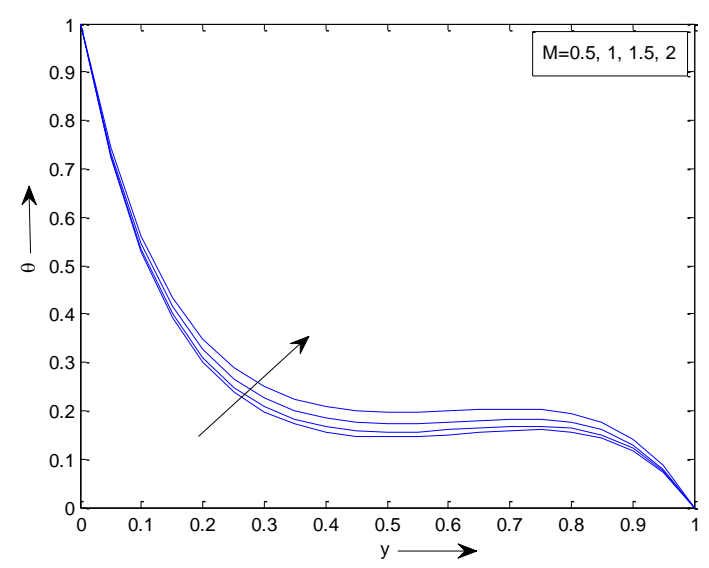

Fig.9: Temperature profile for different $M$

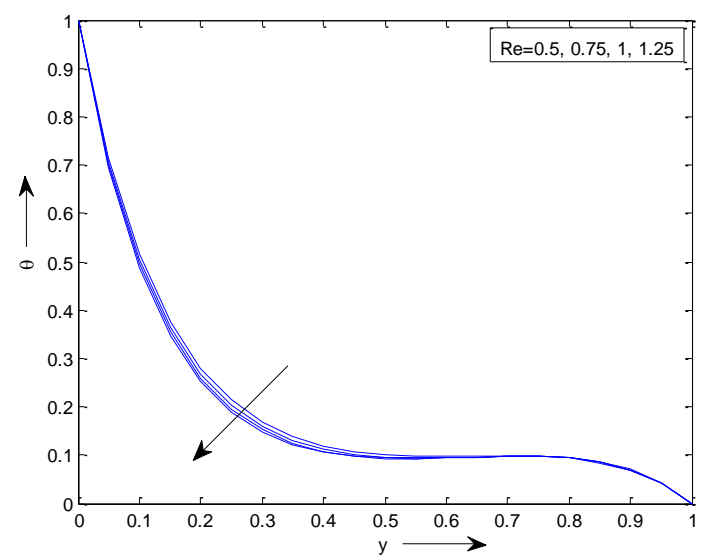

Fig.10: Temperature profile for different $\mathrm{Re}$

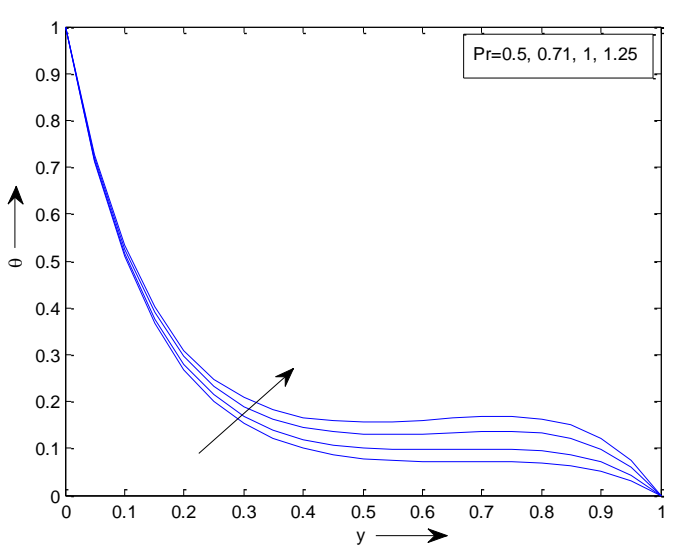

Fig.11: Temperature profile for different $\mathrm{Pr}$ 


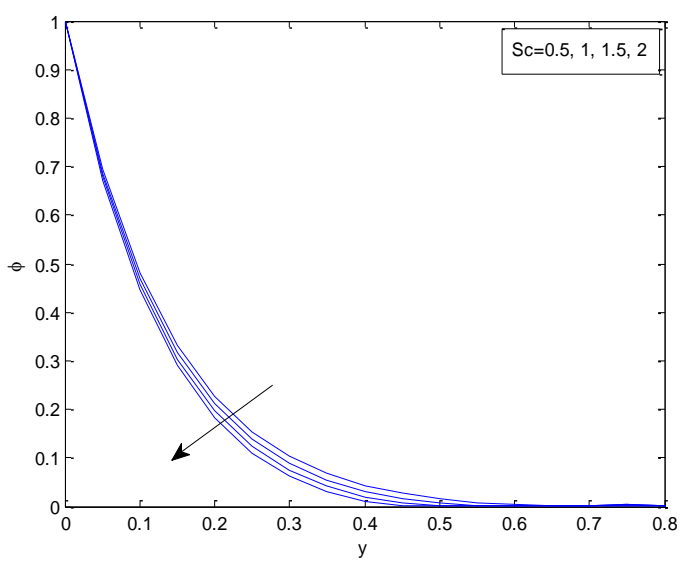

Fig.12: Species Concentration profile for different Sc

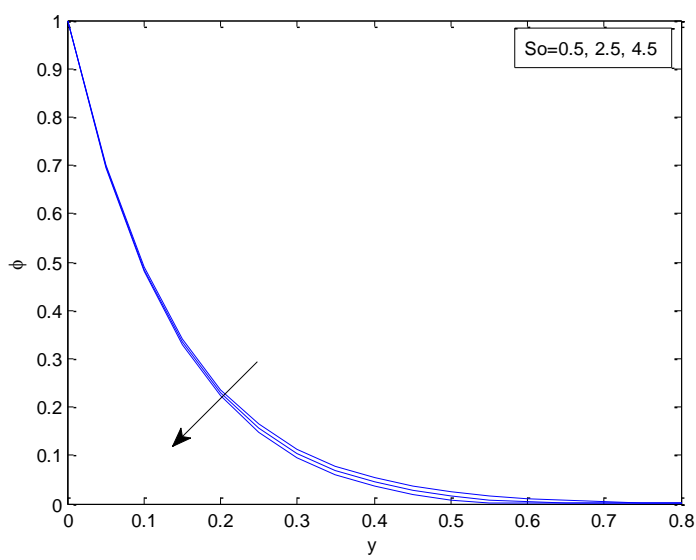

Fig.13: Species Concentration profile for different So
The variation of coefficient of skin-friction $\left(C_{f}\right)$, rate of heat transfer in terms of Nusselt number $(N u)$ and rate of mass transfer in terms of Sherwood number $(S h)$ at the plate $y=0$ against $M, E c, \theta_{r}$ and $\theta_{c}$ are demonstrated from Table-1 to Table-4.

From these tables it is seen that coefficient of skin-friction increases for the increasing values of $\mathrm{M}$ and $\mathrm{Ec}$, while that of decreases for the increasing values of $\theta_{r}$ and $\theta_{c}$.

It is observed that Nusselt number $(\mathrm{Nu})$ enhances due to raise of the thermal conductivity parameter $\theta_{c}$ and viscosity parameter $\theta_{r}$, while it decreases with the increase of the Eckert number $E c$.

Further it is seen that Sherwood number $(S h)$ increases with the increasing values of the thermal conductivity parameter $\theta_{c}$ and Eckert number $E c$. But it decreases with magnetic parameter $M$ and viscosity parameter $\theta_{r}$.

Table 1:

\begin{tabular}{|c|c|c|c|c|c|c|c|c|c|}
\hline \multirow{2}{*}{$\begin{array}{c}\mathbf{M} \rightarrow \\
\Theta_{\mathrm{r}} \downarrow\end{array}$} & \multicolumn{3}{|c|}{0.5} & \multicolumn{3}{c|}{1} & \multicolumn{3}{c|}{1.5} \\
\hline & $\mathrm{Cf}$ & $\mathrm{Nu}$ & $\mathrm{Sh}$ & $\mathrm{Cf}$ & $\mathrm{Nu}$ & $\mathrm{Sh}$ & $\mathrm{Cf}$ & $\mathrm{Nu}$ & Sh \\
\hline 4 & 0.150922 & 0.533161 & 1.359811 & 0.150927 & 0.533158 & 1.359474 & 0.150931 & 0.533156 & 1.359136 \\
\hline 7 & 0.147029 & 0.533201 & 0.83382 & 0.147035 & 0.533199 & 0.833477 & 0.14704 & 0.533198 & 0.833134 \\
\hline 10 & 0.145136 & 0.533225 & 0.558316 & 0.145145 & 0.533225 & 0.557943 & 0.145154 & 0.533224 & 0.55757 \\
\hline 13 & 0.144112 & 0.533242 & 0.386949 & 0.144123 & 0.533242 & 0.386554 & 0.144133 & 0.533241 & 0.386159 \\
\hline 16 & 0.1435 & 0.533254 & 0.269979 & 0.143511 & 0.533254 & 0.269569 & 0.143523 & 0.533254 & 0.269159 \\
\hline
\end{tabular}

Table 2:

\begin{tabular}{|c|c|c|c|c|c|c|c|c|c|}
\hline \multirow{2}{*}{$\begin{array}{c}M \rightarrow \\
\Theta_{c} \downarrow\end{array}$} & \multicolumn{3}{|c|}{0.5} & \multicolumn{3}{c|}{1} & \multicolumn{3}{c|}{1.5} \\
\cline { 2 - 10 } & $\mathrm{Cf}$ & $\mathrm{Nu}$ & $\mathrm{Sh}$ & $\mathrm{Cf}$ & $\mathrm{Nu}$ & $\mathrm{Sh}$ & $\mathrm{Cf}$ & $\mathrm{Nu}$ & Sh \\
\hline 4 & 1.41119 & 0.534138 & 0.178119 & 1.37161 & 0.534134 & 0.175767 & 1.33256 & 0.53413 & 0.173415 \\
\hline 7 & 1.3559 & 0.534141 & 0.209315 & 1.31357 & 0.534137 & 0.206676 & 1.27214 & 0.534133 & 0.204037 \\
\hline 10 & 1.28086 & 0.534143 & 1.316958 & 1.23668 & 0.534139 & 1.314163 & 1.19365 & 0.534135 & 1.311367 \\
\hline 13 & 1.2044 & 0.534145 & 1.576569 & 1.15895 & 0.534141 & 1.573675 & 1.11477 & 0.534137 & 1.570781 \\
\hline 16 & 1.1347 & 0.534146 & 1.75631 & 1.08826 & 0.534142 & 1.753348 & 1.04312 & 0.534138 & 1.750386 \\
\hline
\end{tabular}


Table 3:

\begin{tabular}{|c|c|c|c|c|c|c|c|c|c|}
\hline \multirow{2}{*}{$\begin{array}{c}\text { Ec } \rightarrow \\
\Theta_{\mathrm{r}} \downarrow\end{array}$} & \multicolumn{3}{|c|}{0.05} & \multicolumn{3}{c|}{0.1} & \multicolumn{3}{c|}{0.15} \\
\hline 4 & Cf & $\mathrm{Nu}$ & Sh & Cf & Nu & Sh & Cf & Nu & Sh \\
\hline 4 & 0.150245 & 0.533161 & 1.011396 & 0.15025 & 0.533161 & 1.35846 & 0.150255 & 0.53316 & 1.807293 \\
\hline 7 & 0.147071 & 0.533201 & 0.596549 & 0.147069 & 0.533201 & 0.832447 & 0.147068 & 0.533201 & 1.147794 \\
\hline 10 & 0.145557 & 0.533225 & 0.353637 & 0.145555 & 0.533225 & 0.556824 & 0.145552 & 0.533225 & 1.06343 \\
\hline 13 & 0.144699 & 0.533242 & 0.190553 & 0.144697 & 0.533242 & 0.38537 & 0.144696 & 0.533242 & 0.950722 \\
\hline 16 & 0.14416 & 0.533254 & 0.0746 & 0.144159 & 0.533254 & 0.268341 & 0.144157 & 0.533254 & 0.855002 \\
\hline
\end{tabular}

Table 4:

\begin{tabular}{|c|c|c|c|c|c|c|c|c|c|}
\hline \multirow{2}{*}{$\begin{array}{c}\text { Ec } \rightarrow \\
\Theta_{c} \downarrow\end{array}$} & \multicolumn{3}{|c|}{0.05} & \multicolumn{3}{c|}{0.1} & \multicolumn{3}{c|}{0.15} \\
\cline { 2 - 9 } & Cf & $\mathrm{Nu}$ & Sh & Cf & Nu & Sh & Cf & Nu & Sh \\
\hline 4 & -0.07164 & 0.534136 & 1.460844 & -0.08405 & 0.534138 & 1.533446 & -0.08946 & 0.53414 & 1.635338 \\
\hline 7 & -0.10557 & 0.534139 & 1.602239 & -0.10892 & 0.534141 & 1.687625 & -0.10911 & 0.534143 & 1.80794 \\
\hline 10 & -0.12155 & 0.534141 & 1.672856 & -0.12099 & 0.534143 & 1.765265 & -0.1194 & 0.534145 & 1.895767 \\
\hline 13 & -0.12925 & 0.534142 & 1.715216 & -0.12726 & 0.534145 & 1.812054 & -0.12514 & 0.534147 & 1.949001 \\
\hline 16 & -0.13324 & 0.534144 & 1.743455 & -0.13078 & 0.534146 & 1.843338 & -0.12847 & 0.534148 & 1.984726 \\
\hline
\end{tabular}

\section{CONCLUSIONS}

The investigation leads to the following conclusions:

The fluid motion is retarded for the increase of viscosity and the application of transverse magnetic field.

$>\quad$ Dissipation enhances the velocity.

$>\quad$ Viscosity decrease the temperature whereas conductivity enhances it.

The wall shear stress of fluid decreases with increase of thermal conductivity as well as viscosity.

Magnetic field minimizes the viscous drag on the plate.

The rate of heat transfer increases with increasing viscosity and thermal conductivity. But it decreases with magnetic field.

$>\quad$ Rate of mass transfer at the plate are considerably increased due to increasing thermal conductivity, while it decreases with increasing viscosity and magnetic field.

\section{REFERENCES}

[1] Kim Y J., "Unsteady MHD convective heat transfer past a semi infinite vertical porous moving plate with variable suction", Int. J Eng. Sc,.38, 833-845(2000).

[2] Hazarika G.C. , "Variable viscosity and thermal conductivity on MHD rotating fluid with dissipation and chemical reaction", American journal of Mathematics and Mathematical Sciences.

[3] Ahmed N., Sarmah H. K., and Kalita D., "Thermal diffusion effect on a 3-dimensional free convection with mass transfer flow from a porous vertical plate", Lat. Am.Appl.Res.41

[4] Sharma P.R. and Mathur P.R., "Steady laminar free convection flow of an electrically conducting fluid along a porous hot vertical plate in the presence of a heat source/sink", Indian J .pure appl.Math.26(11) (1995) 1125-1134.

[5] M Anwar Hussain, K, Khanafer and K Vafai, The effect on radiation on free convection flow of fluid with variable viscosity from a porous vertical plate. Int. J Therm. Sc.-40(2001) 115-124

[6] Seth G.S., Ansari M.S. and Nandkeolyar R. "MHD natural convection flow with radiative heat transfer past an impulsively moving plate with ramped wall temperature", Heat Mass Transf.47. 551-561(2011).

[7] Lai, F. C. and Kulacki, F.A.(1991), "The effect of variable viscosity on convective heat and mass transfer along a vertical surface in saturated porous media", Int. J. of Heat and Mass Transfer, Vol. 33, pp. 1028-1031.

[8] Hazarika G.C. and Khound P.K. (2000), "The effect of variable viscosity and thermal conductivity on liquid film on an unsteady stretching surface", Proc. Of $46^{\text {th }}$ Annual Tech. Session, Ass. Sc. Soc., pp.47-56. 